\title{
THE POWER LINE HEIGHT ANALYSIS CHART DRAWING BASE ON MAPMATRIX
}

\author{
He YuanRong ${ }^{1,2, *}$, Ma WeiWei ${ }^{1}$, Wang XiaoRong ${ }^{1}$, Dai JiaQuan ${ }^{1}$, Zheng JieLong ${ }^{1}$ \\ ${ }^{1}$ College of Computer and Information Engineering, Xiamen University of Technology, Xiamen, China - 2012112001@xmut.edu.cn, \\ (446903707, 980753539, 842455412,654516459)@qq.com, \\ ${ }^{2}$ Key Laboratory of Ecological Environment and Information Atlas Fujian Provincial University, Putian University, Putian, China - \\ 2012112001@xmut.edu.cn
}

Commission VI, WG VI/4

KEY WORDS: UAV remote sensing, MapMatrix, Power line height, Stereo mapping

\begin{abstract}
:
The power patrol has been completed by manual field investigation, which is inefficient, costly and unsafe. In order to extract the height of the power line and its surrounding ground objects more quickly and conveniently, and better service for power line patrol. This paper uses remote sensing data of unmanned aerial vehicle to carry out aerial triangulation, stereo model establishment and binocular stereo vision height extraction base on MapMatrix software, then obtains the power line height analysis chart. Then LiDAR point cloud data is used to verify the accuracy of the power line height analysis chart. The results show that this method not only meets the standard of power line patrol, but also improves the efficiency and quality of power line patrol.
\end{abstract}

\section{INTRODUCTION}

\subsection{General Instructions}

Based on the continuous development of China's economy at this stage, and the increasing demand for electricity in life and industry, the development of China's power system is in full swing. The scale of national power generation has been steadily always ranked the first in the world, and it has been also created a huge power transmission network (China Electricity Council, 2019). $80 \%$ to $90 \%$ of the transmission lines in China are between the mountains. At the same time, the lines are mostly non-insulated aluminum stranded wires. When there are branches in the power corridor that touch the power line, it will cause single-phase grounding, short-circuit or circuit breaking faults, resulting in power electric accident (Geng, 2011). In the case of unattended transmission lines with large coverage, long line distances and high safety and reliability requirements, it is highly probable that power failures caused by vegetation disturbances in the power corridor may occur due to untimely inspections (Wei et al, 2018). According to the US West Power Company report, there were 37 cases of power accidents caused by tree barriers in the power corridor in 2007 (North American electric reliability corporation, 2007). In the first half of 2012 , the power grid trip caused by tree barriers in Guangdong Province increased by $150 \%$. As of the end of August of that year, the whole province had cleaned up 1,287 tree barriers and illegal buildings with lines of $110 \mathrm{kV}$ and above (Guangdong Power Grid Corporation, 2012). Due to the large-scale power outage caused by such events and its economic losses, the power line patrol work has become indispensable. At the same time, it is necessary to improve the accuracy of distance detection of the patrol, so as to reduce the power accidents caused by the small safety distance of the power corridor.

Traditional methods of overhead power line patrol include field surveys and aerial surveys. The core approach has remained the same for decades. Specifically, it is a ground trek survey or an aerial survey by helicopter. The investigation team is subject to the situation, mainly depending on the cost, type of problem and priority of inspection. Ground-based field surveys have high labor intensity, low inspection efficiency, and poor coverage. However, due to their long-term resident inspection can improve the problem detection rate. Although the helicopter aerial survey has high efficiency and limited restriction, its speed detection rate is greatly reduced due to its relatively fast speed and limited crew observation ability (Matikainen et al, 2016). Both methods are based on human visual observation, and there is no reliable data guarantee. If the distance measurement between the power lines and the surrounding ground objects is measured by using an insulating tool, although the credibility can be increased, it is not suitable for application because of its safety hazards.

Unmanned aerial vehicles (UAV), also known as unmanned aerial systems (UAS), are an emerging field with multiple potential applications and development prospects (Colomina et al,2014). The use of UAV for power line patrol of overhead power lines is a hot research topic at present, which involves aviation, communication, image recognition, surveying and mapping, and other professional fields, but also because of its low cost, good maneuverability, adaptability and simple operation. And so on, so that it has more important use value in electric power patrol and broad application prospect of (Chen et al, 2008). China is also exploring the patrol mode of "unmanned aerial vehicle patrol mode mainly and manual patrol mode as auxiliary".

In recent years, the number of research articles on UAV power line has increased significantly. Foreign Jozkow G uses the images acquired by the UAV to build a three-dimensional model of the power lines, and uses dense images matching to generate point clouds, so that the three-dimensional modeling of the wire has a catenary curve. This method requires the image to have a high degree of overlap and appropriate resolution (Jozkow et al, 2015). Using the UAV laser scanner data,

\footnotetext{
* Corresponding author

E-mail address: 2012112001@xmut.edu.cn (He YuanRong).
} 
Wallace LO extracted $98 \%$ of the trees correctly from the point cloud. The positioning accuracy of the trees reached 0.48 meters and the accuracy of the canopy area was 3.3 square meters (Wallace et al, 2014). Domestic Zhang et al. used LiDAR technology to extract power lines and further studied their various applications (Zhang et al, 2017). Zhang uses aerial images to do relevant researches. Ratio operator is used to extract points on power lines. Modified HT and global Hough operators are used to extract power lines, and the extraction accuracy of power lines can reach $90 \%$ (Zhang, 2012). Zheng et al. used two digital cameras to take aerial photos of the power line, so as to measure the distance between the power line and the ground objects below (Zheng et al, 2010).

In this context, this research uses UAV to obtain highresolution visible light image acquisition of overhead power lines. Based on the aerospace vision development software MapMatrix, one-stop aerial triangulation and stereo model establishment of acquired images are performed. The power line and its ground in the power corridor are highly extracted, and the acquired height information is evaluated and analyzed. Finally, the production of the power line net height analysis map is completed. Compared with laser-point cloud data, although it can obtain high-precision range detection, it has the disadvantages such as high cost, high difficult operation and long processing cycle. This research not only meets the requirements of power patrol line height, but also has high detection efficiency, low maintenance cost, low risk and strong maneuverability. At the same time, the power line net height analysis chart can provide reference for the later power line.

\section{OVERVIEW OF THE RESEARCH AREA}

Fujian Province. The line is located in Xiamen University of Technology, along with Haixiang Avenue. Figure 1 shows the selected UAV image of the research area. The altitude difference is small and the terrain is flat, which ensures flight safety. At the same time, the area contains roads, houses, trees, street lamps and other ground objects.

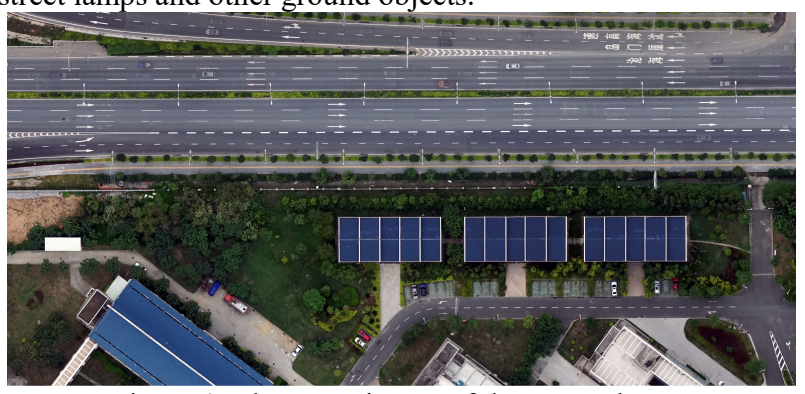

Figure 1. The UAV image of the research area

\subsection{Regional Data Acquisition}

In this research, the eBee UAV system was used to acquire data from the research area. A total of one flight was taken, and the high-resolution UAV remote sensing image acquired was about $0.5 \mathrm{~km}^{2}$. In the aerial photography process, the altitude is about 220 meters and the ground resolution of the image is about $5 \mathrm{~cm} / \mathrm{px}$. In order to improve the quality of the later data processing, the heading overlap rate and the side overlap degree are set to $75 \%$.

After the aerial photography of UAV is completed, it is necessary to check the image data of aerial photography, mainly checking the image overlap degree, cloud haze degree, heading angle, and inclination angle and so on. Finally obtained camera parameter data, partial POS data, image data and control point

\begin{tabular}{|c|c|c|c|c|c|c|}
\hline Image Name & Latitude & Longitude & Altitude & Omg & Pai & Kpa \\
\hline DSC00281.JPG & 24.63163831 & 118.0826249 & 222.733418 & -1.393141 & 1.706413 & -34.822614 \\
\hline DSC00282.JPG & 24.63144033 & 118.0823899 & 221.789116 & -3.807522 & -0.283897 & -31.292293 \\
\hline DSC00283.JPG & 24.63124566 & 118.0821566 & 222.418575 & -5.125986 & 1.371959 & -33.558960 \\
\hline DSC00284.JPG & 24.63104805 & 118.0819111 & 222.745673 & -2.479097 & 1.601916 & -31.441847 \\
\hline DSC00285.JPG & 24.63084755 & 118.0816759 & 222.645995 & -1.920404 & -0.827469 & -34.043496 \\
\hline DSC00286.JPG & 24.63065697 & 118.0814503 & 223.379017 & -5.801280 & -0.152461 & -34.663338 \\
\hline DSC00287.JPG & 24.63045335 & 118.0812057 & 224.491747 & -1.881941 & -2.051476 & -31.070978 \\
\hline DSC00288.JPG & 24.63024477 & 118.0809552 & 223.533688 & -0.779918 & -1.275157 & -35.594255 \\
\hline DSC00289.JPG & 24.63006031 & 118.0807311 & 222.980274 & -2.212301 & -0.335420 & -30.776431 \\
\hline DSC00290.JPG & 24.62986471 & 118.0805057 & 222.975961 & -3.087439 & -0.072524 & -33.384581 \\
\hline
\end{tabular}

\subsection{Introduction to The Research Area}

data are shown as follows:

This research selected $220 \mathrm{KV}$ high-voltage AC transmission line corridor in the first section of Jimei District, Xiamen City,

Table 1. POS data comparison table

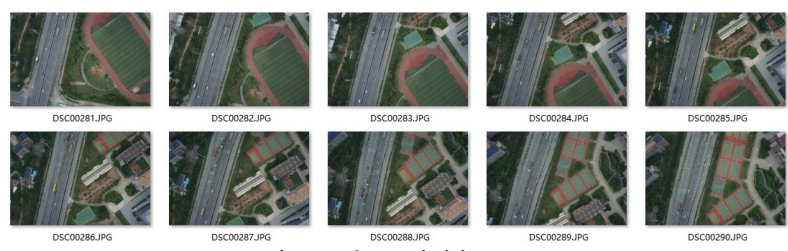

Figure 2. aerial image

\section{RESEARCH METHOD}

\subsection{Double Image Stereo Mapping Principle}

Double-image stereo mapping refers to reconstructing of the ground solid geometric model by using a stereo image pair (taking two photos with overlapping images of the same ground scene at two shooting stations), and measuring of the geometric model directly. Give a topographic map that meets the specified scale or establish a digital ground model.

Figure 3 (a) shows the shooting process from the air to the ground, S1 and S2 are two camera stations (camera objective lens center), two images $\mathrm{P} 1$ and $\mathrm{P} 2$ are taken, the spatial distance between $\mathrm{S} 1$ and $\mathrm{S} 2$ is called the photography baseline $\mathrm{M}$, the ground points $\mathrm{A}, \mathrm{B}, \mathrm{C}, \mathrm{D}$, etc.emitted the light, through $\mathrm{S} 1$ and $\mathrm{S} 2$, is respectively constrained in the overlapping range of the images on the P1 and P2 images, and becomes two photographic beams. Light rays $\mathrm{AS}_{1}, \mathrm{AS}_{2}, \mathrm{CS}_{1}$ and $\mathrm{CS}_{2}$, etc. are all corresponding light rays of the same name. At this time, the light of the same name and the baseline are always in same plane, that is, the three vectors $\mathrm{S}_{1} \mathrm{~S}_{2}, \mathrm{~S} 1 \mathrm{~A}$ and $\mathrm{S}_{2} \mathrm{~A}$ are coplanar, which is also known as the light rays of the same name intersecting in pairs. According to the reversibility of the 
photography process, two projectors designed like cameras are designed to mount the images $\mathrm{P} 1$ and $\mathrm{P} 2$ into the two projectors respectively and keep the orientation of the two projectors the same as the orientation of the camera during photography, but the distance between the objective lenses. Zoom out, that is, the projector moves from $\mathrm{S}_{2}$ to $\mathrm{S}_{2}$ (as shown in Fig. 3(b)). At this time, the distance between the two projectors for $\mathrm{S}_{1} \mathrm{~S}^{\prime} 2=\mathrm{m}$, called $\mathrm{m}$ projection baseline. On the projector, with the spotlight illumination, all the light of the same name in the two projectors in the east of the two sides can still form the intersection of the space and the intersection of $\mathrm{A}^{\prime}$, $\mathrm{B}^{\prime}, \mathrm{C}^{\prime}, \mathrm{D}^{\prime}$ and so on. The collection of all these intersection points constitutes a geometric model similar to the ground. The scale of the model is $1: \mathrm{p}=\mathrm{m}: \mathrm{M}$. This process is called the geometric inversion of the photographic process, which is the basic principle of stereoscopic photogrammetry.
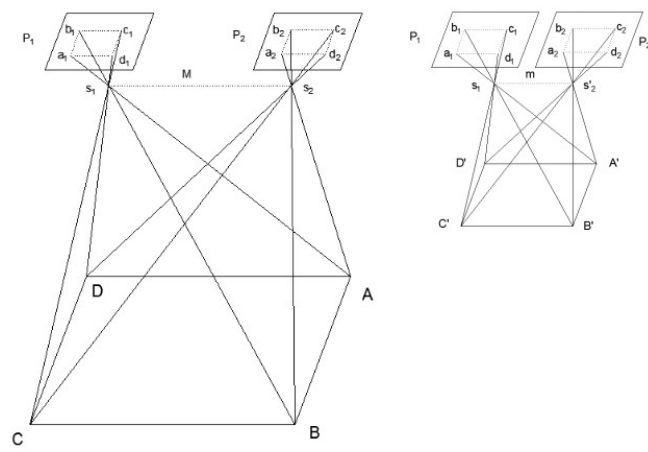

(a) Photography process

(b) Geometric reversal Figure 3. Dual image stereo mapping principle

\subsection{Power Line and Feature Height Point Extraction Process}

Any ground point in the overlapping range of two images of a stereo image has its namesake image points on two images respectively, and forms the namesake ray with the corresponding photography center. The photographic rays of the namesake intersect each other. Therefore, there is a fixed geometric relationship between the eponymous image point and the ground point in the photography baseline. To perform stereoscopic mapping with image pairs, it is necessary to reconstruct a geometric model similar to the field and conform to the scale and spatial orientation.If the internal and external orientation elements of image pairs can be restored, the abovementioned inherent geometric relationship can be restored, so the process of $3 \mathrm{D}$ reconstruction and the elevation point acquisition of power line is as follows:

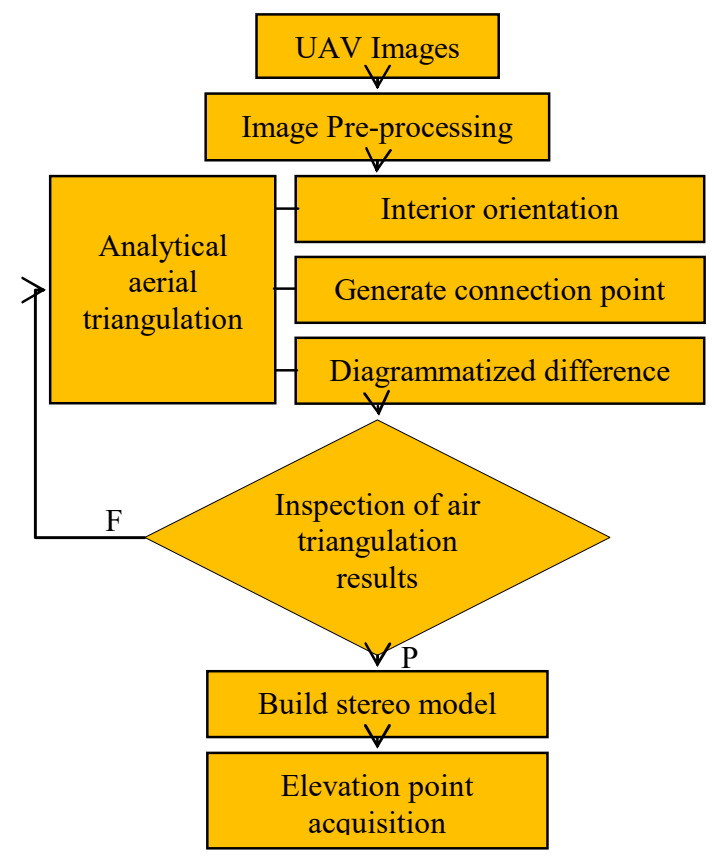

Figure 4. Height extraction flow chart

\section{POWER LINE AND FEATURE HEIGHT EXTRACTION}

\subsection{Analytical Aerial Triangulation}

\subsubsection{Inner Orientation}

The internal orientation is to restore the relative position of the photo and the camera according to the frame mark coordinates of the photo and the corresponding camera calibration parameters, that is, to establish the photo coordinate system (Chen et al, 2004). It is necessary to set the scanning resolution and camera parameters of the image before performing the internal determination. For digital images, the HAT module of MapMatrix can be used for fully automatic internal orientation, which will automatically refresh the coordinates of the image at the same time. After the execution, the blue projection center mark will be displayed in the middle of the image with successful internal orientation, as shown in Figure 5. For film images, after performing the "automatic internal orientation" operation, if the automatically calibrated point is not in the center of the frame label, manual intervention is required to perform internal orientation editing correction.

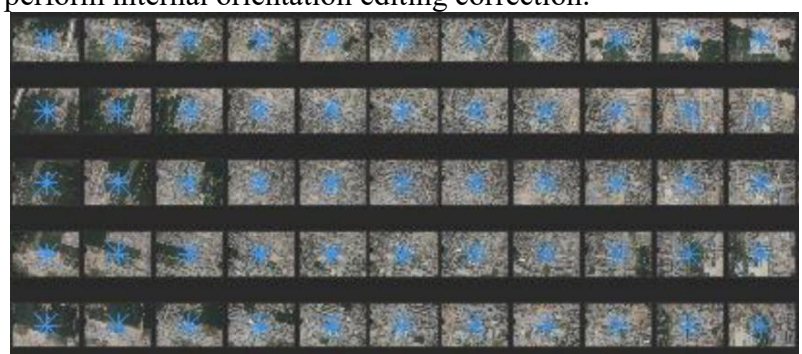

Figure 5. Internal orientation results

\subsubsection{Relative Orientation}

When two cameras pick up a stereo pair on the same ground, the two light rays of the same name in any pair of stereo pairs intersect at the object point, that is, there is a phenomenon that the pair of light rays of the same name intersect, if two images are kept The relative position and attitude relationship are unchanged, the two images are moved, rotated and the length 
of the baseline is changed as a whole, and the characteristics of the pair of light rays of the same name do not change. The relative orientation of the analytical method is based on the internal geometric relationship of stereoscopic image pairs of the same name. Through the measured image point coordinates, the relative orientation elements are calculated by analytical calculation, and a stereo model similar to the ground is established to determine the three-dimensional coordinates of model points. The relative orientation is independent of the absolute position of the photo, and no ground control points are required.

The purpose of relative orientation is to establish a threedimensional model. The operation process of the empty three mainly depends on the relative orientation, and its quality directly affects the result of absolute orientation, which in turn affects the effect of stereo.

\subsubsection{Connection Point Generation and Screening}

Connection points are image points of the same name used to connect adjacent models. The ground coordinates of the connection points are unknown, but can be identified and measured in overlapping areas on two or more images. The connection points should also be evenly distributed over the image of the area, most typically 9 connection points per image. In a survey area, if there is $60 \%$ of the headings overlap and $30 \%$ of the sideways overlap, 9 connection points on each image are enough to connect the entire navigation belt and achieve better accuracy (Qian, 2012). As shown in Figure 6:

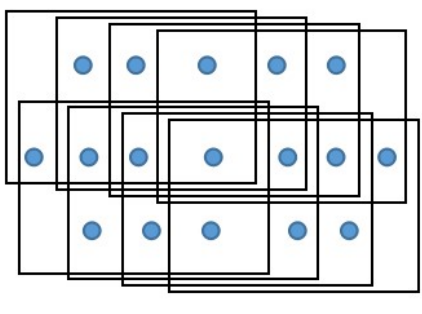

Connection Point

Figure 6. Distribution of connection points

According to the internal and external orientation elements, camera parameters, control points and other data of the navigation belt, the connection points of the images are automatically extracted, and the generated connection points are as shown in Figure. 7:
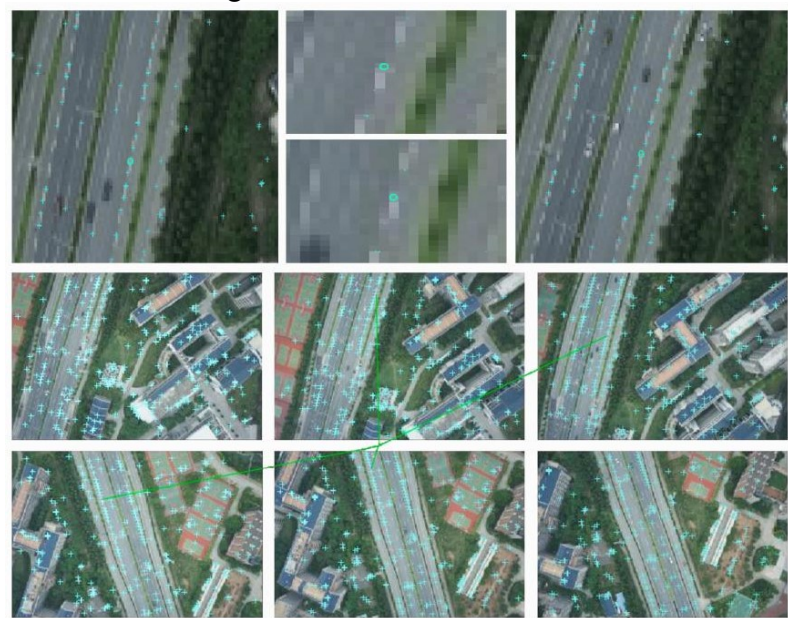

Figure 7. Generated connection points

After the connection points are generated, the bad points and the error points in the connection point need to be filtered and removed, and mainly eliminating the points with overlap degree less than 2 and gross difference value of comprehensive Max. As shown in Figure 8, is the dispute point window, from which the unnecessary connection points are removed, so that it does not participate in the null three operation and affect the accuracy of the result.

\begin{tabular}{|l|c|c|c|c|c|c|}
\hline Issues View & \multicolumn{1}{|c|}{ Type } & Overlap & Max & Rx_Max & Ry_Max \\
\hline$\square 1$ & 100000334 & connect. & 4 & $1986.31 \ldots$ & $1986 \ldots$ & $414.3 \ldots$ \\
\hline$\square 2$ & 100002902 & connect. & 3 & $1456.75 \ldots$ & 1.367123 & $1456 \ldots$ \\
\hline$\square 3$ & 100000112 & connect. & 5 & $1421.60 \ldots$ & $1421 \ldots$ & $-699 \ldots$ \\
\hline$\square 4$ & 100002075 & connect. & 5 & $1379.56 \ldots$ & $-271 \ldots$ & $1379 \ldots$ \\
\hline$\square 5$ & 100002841 & connect. & 3 & $1307.02 \ldots$ & $-304 \ldots$ & $-1307 \ldots$ \\
\hline$\square 6$ & 100002072 & connect. & 5 & $1293.20 \ldots$ & $124.1 \ldots$ & $1293 \ldots$ \\
\hline$\square 7$ & 100000460 & connect. & 5 & $1270.28 \ldots$ & $79.09 \ldots$ & $1270 \ldots$ \\
\hline$\square 8$ & 100000537 & connect. & 5 & $1214.99 \ldots$ & $72.93 \ldots$ & $-1214 \ldots$ \\
\hline$\square 9$ & 100000532 & connect. & 5 & $1207.33 \ldots$ & $497.1 \ldots$ & $-1207 \ldots$ \\
\hline$\square 10$ & 100001203 & connect. & 3 & $1204.22 \ldots$ & $78.31 \ldots$ & $-1204 \ldots$ \\
\hline$\square 11$ & 100001520 & connect. & 4 & $1180.02 \ldots$ & $108.6 \ldots$ & $1180 \ldots$ \\
\hline$\square 12$ & 100002745 & connect. & 3 & $1156.71 \ldots$ & $-129 \ldots$ & $1156 \ldots$ \\
\hline$\square 13$ & 100000572 & connect. & 5 & $1145.71 \ldots$ & $290.3 \ldots$ & $1145 \ldots$ \\
\hline$\square 14$ & 100001837 & connect. & 3 & $1140.28 \ldots$ & $-17.6 \ldots$ & $1140 \ldots$ \\
\hline
\end{tabular}

Figure 8. Screening of the connection points

\subsubsection{Adjustment Calculation}

The adjustment calculation is based on the mathematical model, using a few control points measured in the field, the adjustment calculation (encryption) all the control points (called to be fixed points or to be encrypted points) of required for the photogrammetric operation and the external orientation elements of each photo. The airstrip method, the independent model method and the beam method are the more commonly used adjustment models. They are also classified according to the encryption area, and can be divided into single airstrip method and a regional network method. In this research, the PATB adjustment software is used for the adjustment calculation. It uses the self-checking beam adjustment algorithm which is the most rigorous in theory and can compensate the systematic error. At the same time, it adds advanced and practical gross error detection algorithm, so it can get high accurate adjustment results with efficient gross error detection.

After the connection points are generated automatically, several control points can be punctually inserted around the four corners of the engineering area (at least three ID control points when POS information is not available), and accurate marks should be made on as many relevant images as possible when using HAT module to insert control points.

After inserting the control point, the adjustment can be calculated first to understand the precision of the connection point. If the adjustment is convergent, it is convenient to add other control points, and the edit deletes the coarse points. After the PATB adjustment calculation is solved once, the previously solved sigma value needs to be set to be solved again until the sigma values solved are the same as the red box value in Figure 9. Among them, the blue box is the weight value of the control point. The smaller the value given, the larger the weight value represented, and vice versa. After the adjustment is calculated, the dispute point is edited and then the adjustment calculation is usually performed until there is no obvious dispute point information. In addition, it is necessary to check the "output posterior variance" With calculation of a posteriori variances by inversion " in the Features in the last adjustment calculation. At the same time, the check" With automatic error detection "can be unchecked when there is no obvious big mistake point at the connection point and it is sure that the control point has no problem. 


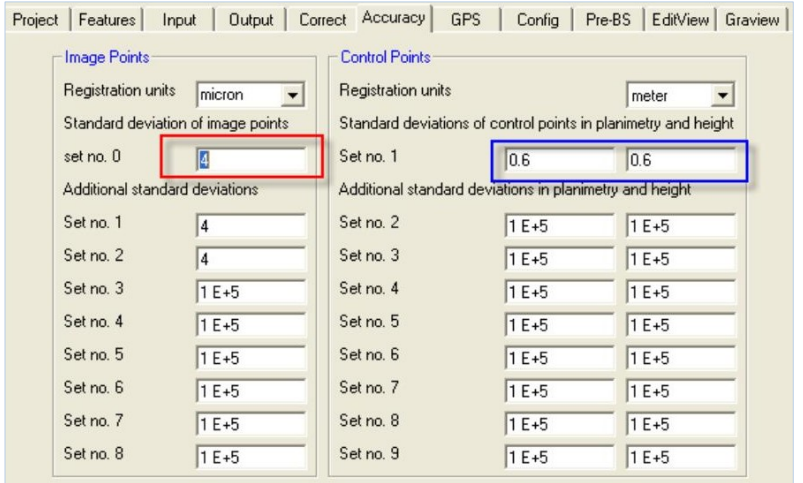

Figure 9. PATB adjustment parameter settings

As shown in Figure 10, it is the *.pri file generated after the adjustment calculation, that is, a PATB calculation report, which includes the calculation precision, whether to warn and error information, etc. We can see the adjustment calculation accuracy by looking at the rx, ry, rz in the figure. Thus we can judge whether the accuracy meets the requirements of the operation.

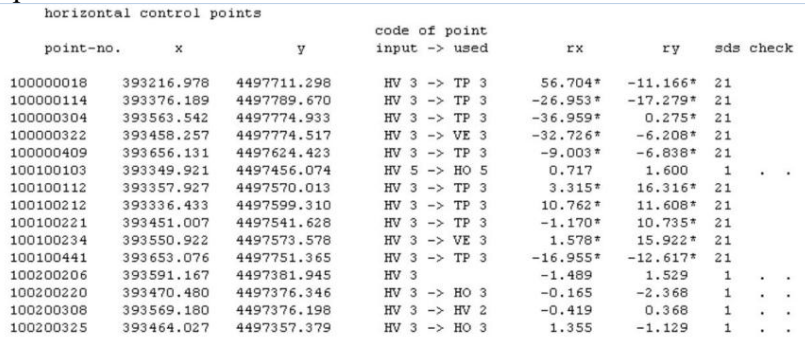

Figure 10. PATB solution report

\subsection{Restore stereo Model}

After the aerial triangulation is completed, the MapMatrix project file can be output, the created project file is opened in the MapMatrix main page, a stereo image pair and a DLG project file are created, and the created stereo image pair model into the DLG project file. Digitize the DLG project file to open the Feature One module and set the graphics scale to 500. For the area where the map is to be taken, open the real-time coreline image of the pair of images to start the elevation point acquisition, as shown in Figure 11.

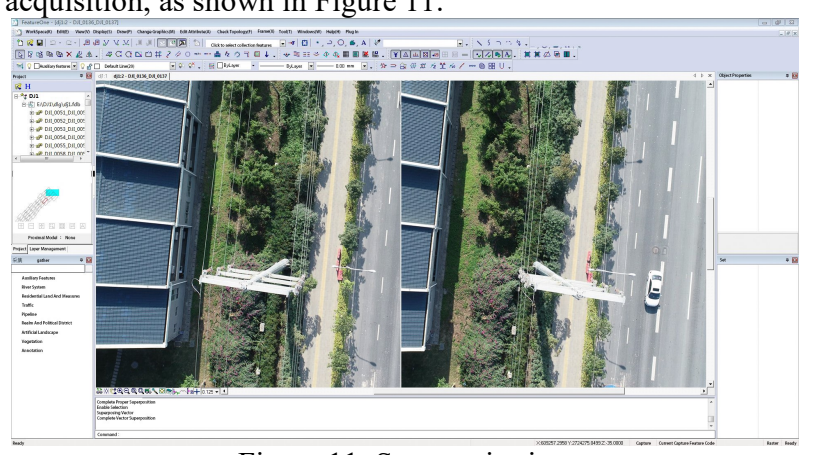

Figure 11. Stereopair view

\subsection{Height Extraction of Power Lines and Ground Features}

The stereopair model made above was used to extract the elevation of power transmission lines and ground features. In the process of elevation point acquisition, we need to first observe the physical conditions within 50 meters of the power corridor. We will understand which higher features in the power corridors pose a threat to the power lines, such as trees, bamboo forests, buildings, construction sites, public facilities, etc. Then, the Feature One module of MapMatrix is used to collect the elevation of power lines and dangerous ground objects. The elevation point of the dangerous features is the highest point of the features, and the elevation point of the transmission line is collected every $15 \mathrm{~m}$. The result is shown in figure 12 and 13.

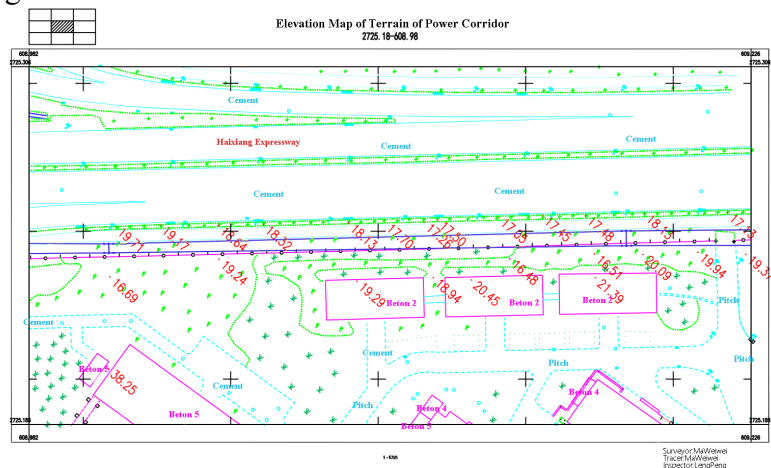

Figure 12. Elevation map of terrain of power corridor

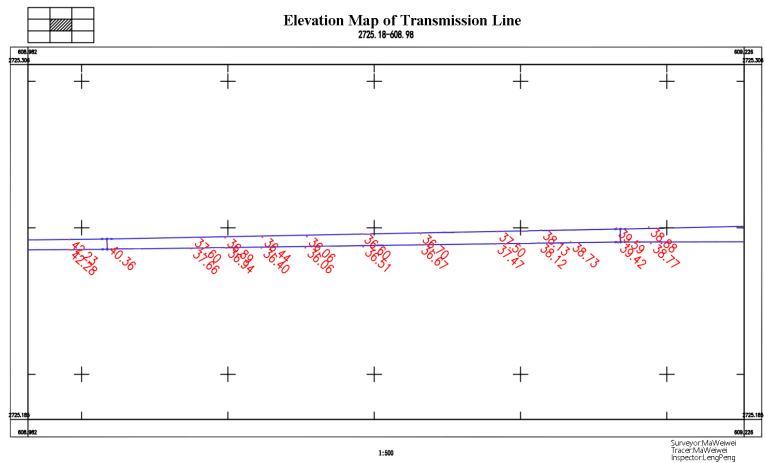

Figure 13. Elevation map of transmission line

It can be known from the elevation map of terrain of power corridor and the elevation map of transmission line:The highest point of the transmission line is in the pole position, with a height of $39.59 \mathrm{~m}$ and $40.36 \mathrm{~m}$ respectively. The lowest position is in the middle position of the two poles, with a height of $36.06 \mathrm{~m}$. The height of the vegetation in the corridor ranges from 16 to 20 meters, and the maximum height of the building is 39.25 meters.

\subsection{Precision Verification}

Although the use of visible light image data for power line extraction will make the data acquisition cost much lower, there is a problem of insufficient spatial positioning accuracy. However, the airborne laser radar system can directly collect high-precision 3D laser point cloud data, including corridor features and towers, hanging line point location, wire sag, etc. The AS - 100 laser measurement and control system using China verifies the accuracy of the experiment, the AS - 100 laser system effectively integrates the scanning system, camera system, the directional positioning system, (including global positioning system (GPS) and inertial navigation device IMU) and the control unit for effective integration, is currently the most advanced field of photogrammetry and remote sensing for earth observation system, the accuracy parameters as shown in Table 2. Using the lidar point cloud data to verify the accuracy of the power lines elevation points collected in this research, 
the data accuracy of this research can be effectively verified within the power line error range.

Table 2. AS-100 Airborne Laser System Parameter Table

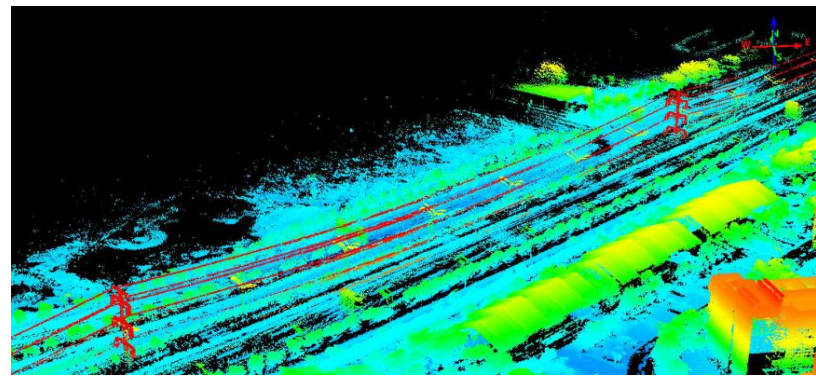

Figure 12. Airborne laser point cloud data

\begin{tabular}{|c|c|c|c|}
\hline Number & $\begin{array}{c}\text { Height from } \\
\text { Experimental } \\
\text { Measurement/m }\end{array}$ & $\begin{array}{c}\text { Height from } \\
\text { Lidar } \\
\text { Measurement/m }\end{array}$ & $\begin{array}{c}\text { Differenc } \\
\text { e Value/m }\end{array}$ \\
\hline 1 & 38.88 & 38.56 & 0.32 \\
\hline 2 & 38.13 & 37.68 & 0.45 \\
\hline 3 & 37.50 & 36.79 & 0.71 \\
\hline 4 & 36.70 & 37.13 & -0.43 \\
\hline 5 & 36.60 & 36.27 & 0.33 \\
\hline 6 & 36.06 & 36.56 & -0.50 \\
\hline 7 & 36.44 & 36.26 & 0.18 \\
\hline 8 & 36.89 & 36.59 & 0.30 \\
\hline 9 & 37.60 & 38.12 & -0.52 \\
\hline 10 & 42.23 & 41.94 & 0.29 \\
\hline
\end{tabular}

As shown in Figure 12, for the laser point cloud data of the research area, a partial comparison data Table 3 is obtained by comparing and extracting the heights of the same position points on the power line.

Table 3. Comparison of measured data

According to the data comparison, the measurement accuracy of this section of the line can reach the sub-meter level, meeting the needs of power line patrol work.

\section{PRODUCTION OF NET HEIGHT ANALYSIS CHART OF POWER LINE}

The danger point is the ground object within a safe distance of the power line. According to the operation rules of overhead transmission lines, the clearance safe distance of transmission lines with different voltages can be known. As shown in the table 4 .

\begin{tabular}{|c|c|c|c|c|c|c|c|}
\hline $\begin{array}{c}\text { Nominal } \\
\text { voltage }(\mathrm{kV})\end{array}$ & 10 & 35 & 110 & 220 & 330 & 500 & 750 \\
\hline $\begin{array}{c}\text { Safe } \\
\text { distance }(\mathrm{m})\end{array}$ & 3 & 4 & 4 & 4.5 & 5.5 & 7 & 8.5 \\
\hline
\end{tabular}

The power line clearance distance can be analyzed by using the elevation map of terrain of power corridor and elevation map of transmission line. Features within the danger zone will be marked. As show in figure 13: The analysis diagram is divided into front view and vertical view. Front view can overlay styles according to the previously measured power line height and ground object height, and vertical view can be superimposed on the topographic map of power corridor, so as to we have a more intuitive understanding of the surrounding environment of power corridor. The red triangle position in the figure is the marked danger point. The position and scope of the danger point can be judged intuitively through these figures. The net height analysis chart can provide a certain reference for the power line patrol staff.

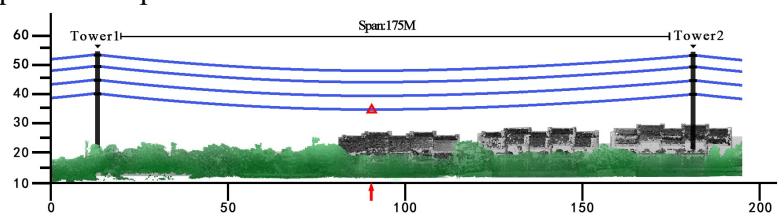

(a) Front view

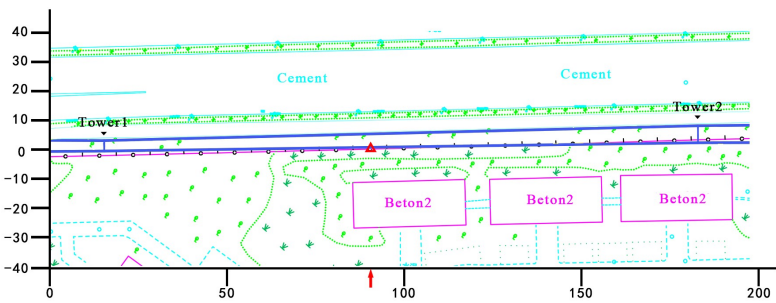

(b) Vertical view

Figure 13. Net height analysis chart of power line

\section{CONCLUSION}

Due to the advantages of flexibility, real-time and low cost of the UAV image acquisition, the UAV remote sensing has been applied more and more widely in power line patrol. In this research, after fully understanding the advantages of UAV remote sensing, HAT module of aerospace perspective MapMatrix mapping software is combined to process the acquired high-resolution images, and Feature One module is used to extract the elevation points of the power lines and the ground objects under the power corridor. Then after using the lidar point cloud data to verify that it meets the power line patrol accuracy requirements, the net height of the power line is analyzed and mapped, and the image data that has a certain reference for the power manager to perform power line management, maintenance, and repair work is not reduced. The difficulty of patrolling the line has improved the efficiency and quality of the power patrol. This research in view of the net height analysis of the current power line height and the height of the ground is carried out, and the power line risk assessment

\begin{tabular}{|c|c|}
\hline Content & Index \\
\hline Laser level & Level 1 \\
\hline Measuring range & $100 \mathrm{~m}$ \\
\hline $\begin{array}{c}\text { Measurement } \\
\text { accuracy }\end{array}$ & $3 \mathrm{~cm}$ \\
\hline Field of view & $\begin{array}{c}360^{\circ} \quad \text { horizontal and } 30^{\circ} \text { vertical }\left(+15^{\circ}\right. \\
\left.\text { to }-15^{\circ}\right)\end{array}$ \\
\hline $\begin{array}{c}\text { Scanning } \\
\text { frequency }\end{array}$ & $5 \sim 20 \mathrm{~Hz}$ \\
\hline $\begin{array}{c}\text { Point cloud } \\
\text { density }\end{array}$ & 300000 points $/ \mathrm{s}$ \\
\hline
\end{tabular}

for the future period is insufficient. If the vegetation of the power corridor is highly classified in the short term, the growth cycle is analyzed. Then, the safety inspection of the power corridor is an important supplement.

\section{ACKNOWLEDGEMENTS}

This research was supported by the Science and Technology Planning Project of Xiamen/Quanzhou City (grant no. 3502Z20183055, 2017G030) and the Open Fund Programs of Key Laboratory of Ecological Environment and Information 
Atlas (Putian University) Fujian Provincial University(Grant No.ST17002).

\section{REFERENCES}

Chen, Shipei., Wang, Tiejun., 2004: Several Problems on Orientation in Digital Photogrammetry. Surveying and Spatial Geography Information 40(2), 19-21.

Chen, Xiaobing., Ma, Yulin., Xu, Zujian., 2008: Discussion on the Inspection Technology of Unmanned Aircraft Transmission Lines. China Southern Power Grid Technology 2(6), 64-66.

China Electricity Council., 2019: China's Power Industry Status and Prospects (2019). China Power Enterprise Management (4), 9.

Colomina, I., Molina, P., 2014: Unmanned Aerial Systems for Photogrammetry and Remote Sensing: a Review. Isprs Journal of Photogrammetry and Remote Sensing 92, 79-97.

Geng, Mingliang., 2011: Talking About The Damage Caused by Trees to $10 \mathrm{kV}$ Overhead Lines. Urban construction theory research: electronic version (22).

Guangdong Power Grid Corporation., 2012: In the first half of 2012, the Guangdong tree barrier caused a power trip accident increased by $150 \%$. http://www.chinairn.com/ News/20120912/174282.html.

Jozkow, G., Vander, jagt, B., Toth, C., 2015: Experiments with Uas Imagery for Automatic Modeling of Power Line 3d Geometry. The International Archives of Photogrammetry, Remote Sensing and Spatial Information Sciences 40(1), 403.

Matikainen, L., Lehtomäki, M., Ahokas, E., et al, 2016: Remote Sensing Methods for Power Line Corridor Surveys. Isprs Journal of Photogrammetry and Remote Sensing, 119, 10-31.

North American electric reliability corporation., 2007: Vegetation-Related Transmission outage report.

Qian, Zunyan., 2012: Research on Key Technology Application of Low Altitude UAV Remote Sensing in Oilfield Measurement. China University of Petroleum (East China).

Wallace, LO., 2014: Development and Application of a Unmanned Aerial Vehicle Laser Scanning System for Forest Management. University of Tasmania.

Wei, Shilei., Xu, Yulin., Wang, Hao., et al, 2018: Research on Power Line Inspection Method Based on UAV Image. Beijing Surveying and Mapping (2), 85-88.

Zhang, Jixian., Duan, Minyan., Lin, Xiangguo., et al, 2017: Comparison and Analysis of 3D Reconstruction Model of Lidar Point Cloud Power Line. Journal of Wuhan University(Information Science Edition) (11), 68-75.

Zhang, Shaoping., 2012: Research on typical target image recognition technology for transmission lines. Nanjing University of Aeronautics and Astronautics.
Zheng, Shunyi., Tan, Jinshi., Ji, Wei., et al, 2010: Research on Power Line Ranging Technology Based on Stereo Camera. Surveying Information and Engineering 35(1), 42-43. 